\title{
Effect of tobacco and nicotine on growth of Haemophilus influenzae in vitro
}

\author{
DAVID ROBERTS AND PETER COLE
}

From the Host Defence Unit, Department of Medicine, Cardiothoracic Institute, Fulham Road, London $S W 36 H P, U K$

SUMMARY Two nutritionally poor bacteriological media, prepared from phosphate-buffered saline and from bronchial secretions, support the growth of Haemophilus influenzae only poorly. The presence of tobacco or pure nicotine in these media stimulates the growth of the organism, and the component(s) responsible appear(s) to be volatile.

The coincident accidental introduction of a small piece of pipe tobacco into a faulty batch of nutrient agar (which poorly supported the growth of Haemophilus influenzae) allowed the effect of the former on growth of the bacterium to be observed and led to experiments to compare this with the effect of nicotine

\section{Material and methods}

\section{BACTERIOLOGICAL MEDIA}

Phosphate-buffered saline agar (PBSA)

Proteose peptone(Oxoid), $10 \mathrm{~g}$, and agar (Oxoid),10g, were dissolved in 1 litre of phosphate-buffered saline (PBS) (Oxoid). After heat sterilisation this was supplemented with $3 \mathrm{mg}$ nicotinamide adenine dinucleotide (NAD) (British Drug Houses-BDH) and $3 \mathrm{mg}$ haemin (BDH), both filter sterilised. The resulting medium reproducibly supported the growth of $\boldsymbol{H}$. influenzae only poorly.

\section{Sputumagar}

A fresh, mucoid specimen of sputum was obtained from a patient with asthma and liquefied using a Dawe Soniprobe (75 watts for 30 seconds). The specimen was centrifuged at $2200 \mathrm{~g}$ for 15 minutes to remove large particles, and $10 \mathrm{ml}$ of the clear supernatant was mixed with an equal volume of molten $3 \%$ agar (Oxoid) in PBS. NAD and haemin were added as before.

TOBACCO AND NICOTINE

St Bruno Rough Cut and pure nicotine (BDH) were used.

Received for publication 21 November 1978
ORGANISM

A non-capsulated strain of $\boldsymbol{H}$. influenzae isolated from a patient with chronic bronchitis was used.

\section{TOBACCO STIMULATION TESTS}

Small pieces of tobacco were placed in the bottom of a petri dish and covered with $20 \mathrm{ml}$ of PBSA. After setting, the plates were flooded with an overnight broth culture of the organism diluted $1 / 100$ in PBS. The excess was drained off, and the plates were dried and incubated for 18 hours at $37^{\circ}$. PBSA plates were also poured, omitting the tobacco from the medium, and seeded with $H$. influenzae as previously. When the plates were dry a small piece of tobacco was applied to the surface of the agar and the plate was incubated for 18 hours at $37^{\circ}$.

An aqueous extract of tobacco was prepared at a concentration of $5 \mathrm{mg}$ per $\mathrm{ml}$ in distilled water and left at room temperature for 1 hour. PBSA plates were poured, the surfaces seeded with organism as before, and dried. Wells were then cut in the agar and filled with the aqueous tobacco extract.

\section{NICOTINE STIMULATION TESTS}

Doubling dilutions of pure nicotine from $0.1 \%$ to $0.03 \%$ were prepared in $10-\mathrm{ml}$ aliquots of molten PBSA and poured into petri dishes. When these had set a top layer of $10 \mathrm{ml}$ of plain PBSA was added. The plates were surface-dried, and a loopful of $H$. influenzae broth culture was spread in a standard manner. Incubation was at $37^{\circ}$ for 18 hours.

Plates containing $20 \mathrm{ml}$ of PBSA were poured and surface seeded with diluted $\boldsymbol{H}$. influenzae broth culture, and a well was cut and filled with a $1 \%$ solution of pure nicotine.

The submerged tobacco and nicotine (in well) 
Fig. 1 Growth of $\mathrm{H}$. influenzae stimulated by tobacco fragment $(\times 10)$.

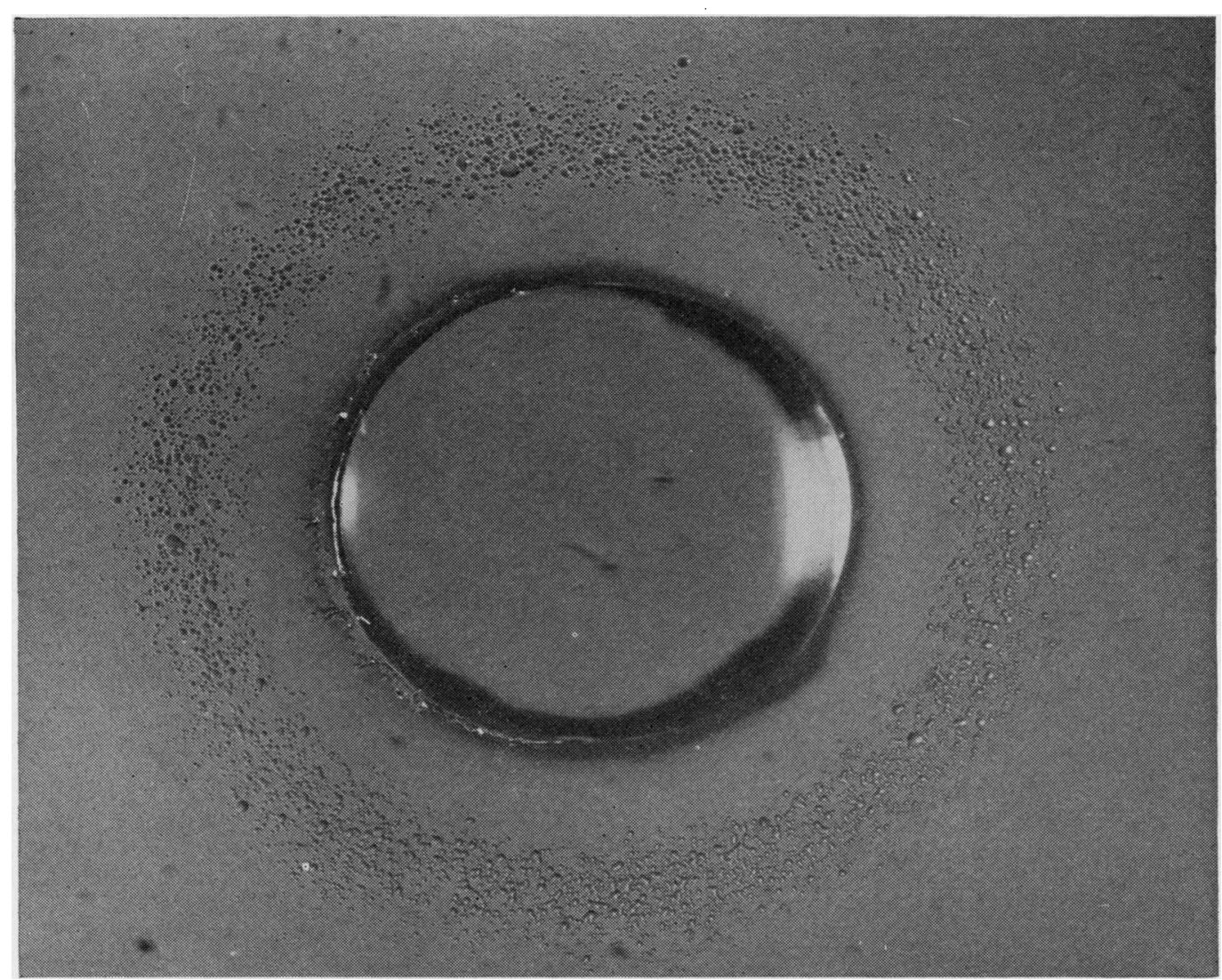

Fig. 2 Growth of $\mathrm{H}$. influenzae around well containing pure aqueous nicotine $(\times 10)$. 


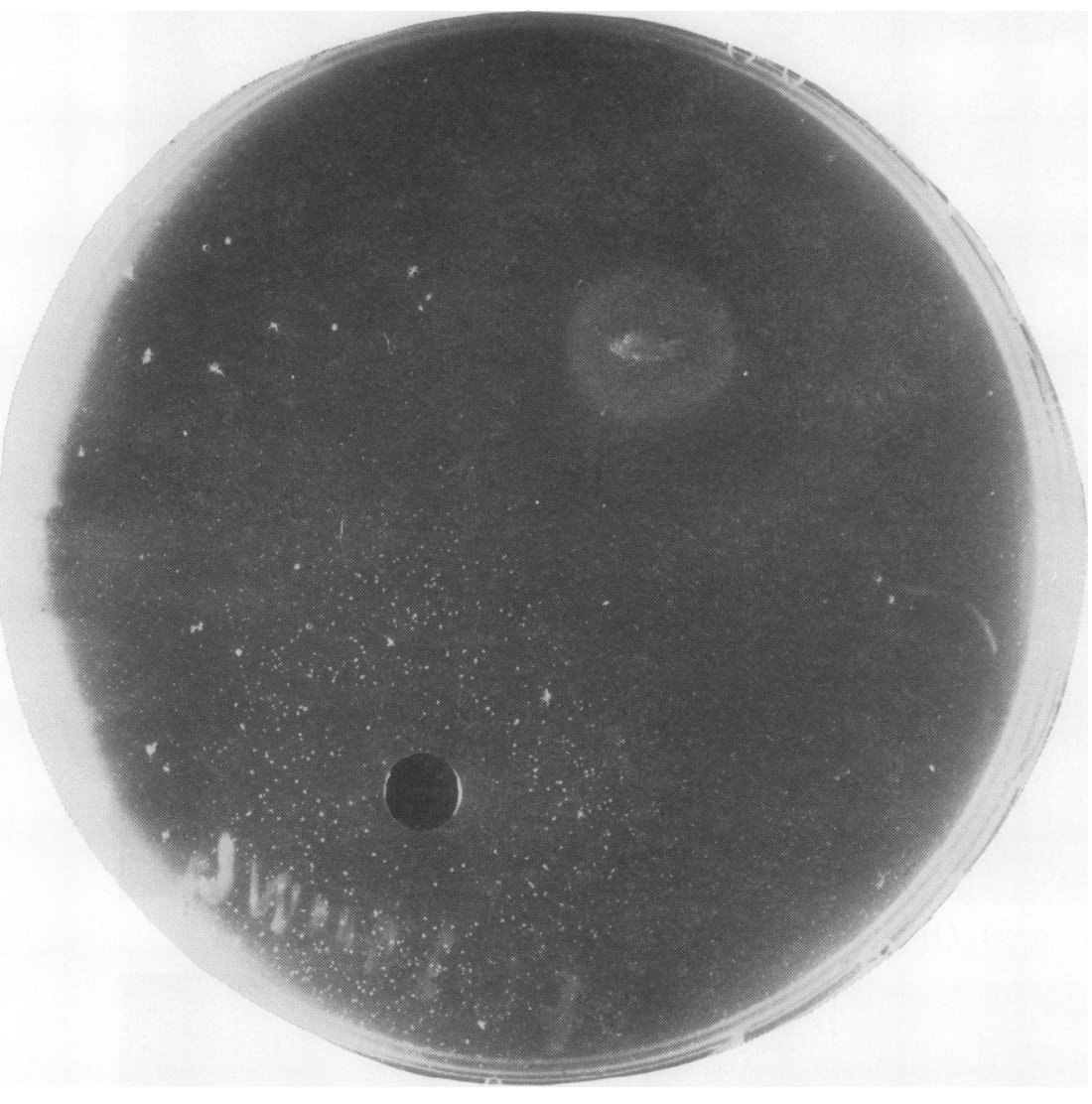

Fig. 3 Growth of $\mathrm{H}$. influenzae on sputum agar $(\times 1 \cdot 25)$.

stimulation tests were repeated using sputum agar, both tests being conducted in the petri dish.

\section{Results}

On the plate with the tobacco incorporated in the medium, prolific growth of the organism was observed directly over the tobacco, the colonies becoming smaller as the distance from the tobacco increased (Fig. 1). The tobacco placed on the surface of the medium gave no stimulation, nor did the aqueous extract of tobacco in the well.

The plate containing $0.1 \%$ nicotine in its bottom layer produced a good growth of $\boldsymbol{H}$. influenzae. Plates containing lower concentrations of nicotine failed to support growth of the organisms.

Where the nicotine had been placed in the well, growth of the organism had occurred around the well but slightly displaced from its edge (Fig. 2).

On the sputum agar plate prolific growth of the organism was observed directly over the tobacco (Fig. 3). Growth of the reference organism on the surface of the plate was not stimulated by pure nicotine in the well, but stimulation of growth of organisms originating from the sputum composing the medium was observed around the well, again slightly separated from the edge of the well (Fig. 3).

\section{Discussion}

The results indicate that tobacco contains a substance which, in very small quantities, can stimulate the growth of $H$. influenzae. The fact that tobacco placed on the surface of the medium, and the aqueous extract in the well, failed to enhance growth suggests that this substance is volatile, possibly nicotine. Pure nicotine in a concentration of $0.1 \%$ at the bottom of the bilayered plate (which would diffuse into an equal volume of agar in the top layer and achieve a final concentration of $0.05 \%$ ) stimulates a prolific growth of $\boldsymbol{H}$. influenzae. Although this concentration is obviously much higher than that produced by a tiny piece of tobacco, a large amount of the nicotine will be lost into the atmosphere of 
the petri dish so that the final concentration in the medium at the end of the culture period is low.

It is now widely accepted that $H$. influenzae is the organism most commonly associated with chronic bronchitis. The presence of antibodies to the organism in the form of specific serum precipitins has been shown to correlate well with the patients' smoking habits and incidence of chest infections (May et al., 1973).

It is not clear whether $H$. influenzae is pathogenic in chronic bronchitis. Current work in this department suggests that bronchial mucosal damage may be caused by toxins produced by the organism. Bronchial secretions have been shown to be a poor culture medium (May and Roberts, 1969), but the experiments described here show that a medium prepared from sputum will support the growth of $H$. influenzae if tobacco or nicotine are added. Therefore it is possible that a regular supplement of nicotine in the bronchial secretions of a heavy smoker may be sufficient to encourage an excessive multiplication of $H$. influenzae. Green and Carolin (1967) have shown that cigarette smoke impairs the antibacterial activity of alveolar macrophages. It is possible that the combination of an increase in the number of organisms due to stimulation of growth by nicotine (thereby producing more toxin) and impaired ability of the pulmonary macrophage to eliminate the organism may lead to increased bronchial mucosal damage in heavy smokers.

We thank Mr K. Moreman, FIIP, FRPS, and the staff of the Photographic Department of the Chester Beatty Research Institute for the photography.

\section{References}

Green, G. M., and Carolin, D. (1967). The depressant effect of cigarette smoke on the in vitro antibacterial activity of alveolar macrophages. New England Journal of Medicine, 276, 421-427.

May, J. R., Peto, R., Tinker, C. M., and Fletcher, C. M. (1973). A study of Hemophilus influenzae precipitins in the serum of working men in relation to smoking habits, bronchial infection and airway obstruction. American Review of Respiratory Disease, 108, 460-468. May, J. R., and Roberts, D. E. (1969). Bronchial infection in cystic fibrosis. Lancet, 1, 602-503.

Requests for reprints to: David E. Roberts, Cardiothoracic Institute, Fulham Road, London SW3 6HP, UK. 